\title{
Evaluation of EGFR mutations in NSCLC with highly sensitive droplet digital PCR assays
}

\author{
XI-WEN JIANG ${ }^{1,2^{*}}$, WEI LIU ${ }^{3^{*}}$, XIAO-YA ZHU ${ }^{1,2}$ and XIAO-XIE XU ${ }^{1,2}$ \\ ${ }^{1}$ Da An Gene Co., Ltd. of Sun Yat-sen University, Guangzhou, Guangdong 510665; \\ ${ }^{2}$ The Medicine and Biological Engineering Technology Research Center of The Ministry of Health, Guangzhou, \\ Guangdong 510080; ${ }^{3}$ Lu He Hospital Capital Medical University Beijing China, Beijing 100069, P.R. China
}

Received August 16, 2018; Accepted February 21, 2019

DOI: $10.3892 / \mathrm{mmr} .2019 .10259$

\begin{abstract}
Targeted drugs have been widely used in the treatment of patients with lung cancer, particularly for those with non-small cell lung cancer (NSCLC). Plasma cell-free DNA is an emerging clinical tool for the detection of epidermal growth factor receptor (EGFR) gene mutation in patients with lung cancer. Detection of circulating tumor (ct) DNA by droplet digital PCR (ddPCR) is a highly sensitive and minimally invasive alternative for the assessment and management of cancer. In the present study, four ddPCR systems were developed to detect the 19DELs, L858R, T790M and C797S mutations of the EGFR gene in plasma ctDNA samples, and all exhibited higher sensitivity compared with the amplification refractory mutation system (ARMS)-PCR assays. The results revealed that the sensitivity of the ddPCR assays for the four major types of EGFR mutant reached $0.04 \%$. In total, 50 plasma ctDNA samples were collected from patients with NSCLC to detect the 19DELs, L858R, T790M and C797S mutations by ddPCR and ARMS-PCR. All the mutations except for C797S were detected and the concordance rates between ddPCR and ARMS-PCR were 96\% (19DELs), 98\% (L858R) and $100 \%(\mathrm{~T} 790 \mathrm{M})$. The fraction of EGFR mutation ranged from 0.43 to $68.07 \%$ using the ddPCR method. Therefore, the present study suggests that the four ddPCR testing systems could be used for early detection of EGFR mutations in plasma samples, so that patients can better select the targeted drugs according to the EGFR mutation.
\end{abstract}

Correspondence to: Dr Xi-Wen Jiang, Da An Gene Co., Ltd. of Sun Yat-sen University, 19 Xiangshan Road, Science Park, High \& New Technology Development District, Guangzhou, Guangdong 510665, P.R. China

E-mail: yuanyecat@vip.sina.com

*Contributed equally

Key words: epidermal growth factor receptor, non-small cell lung cancer, circulating tumor DNA, droplet digital polymerase chain reaction, amplification refractory mutation system-polymerase chain reaction

\section{Introduction}

Lung cancer is the most common type of cancer, with $\sim 326,600$ novel cases and 569,400 mortalities due to lung cancer in China in 2012 (1). Non-small cell lung cancer (NSCLC) is one of the most common types of lung cancer, accounting for $\sim 85 \%$ of lung cancer cases (2). Notably, $75 \%$ of patients are in the middle or advanced stages at the beginning of diagnosis and the 5-year survival rate is very low $(3,4)$. Therefore, the diagnosis of NSCLC is crucial for its treatment.

Molecular studies have identified that the epidermal growth factor receptor (EGFR) gene is a key player in the tumor development of NSCLC (5). EGFR is not only a transmembrane tyrosine kinase receptor, but also a member of the ErbB/HER family (6). Binding of EGFR proteins to other members of the HER family forms homo or heterodimers and determines the autophosphorylation of the intracellular domain and the activation of downstream RAS/RAF/dual specificity mitogen-activated protein kinase kinase mek-2/extracellular signal regulated kinase or phosphatidylinositol 3 kinase/protein kinase B signaling pathways (7). Multiple EGFR mutations have been identified. Most activating mutations of EGFR favor cell survival, proliferation and migration, and metastasis development by increasing the activity of EGFR tyrosine kinase (8). Previous studies have demonstrated that these mutations are in exons 18-21 of the TK domain of EGFR and include either point mutations or in-frame small deletions or insertions $(9,10)$. In unselected Caucasian patients with NSCLC, EGFR mutations are infrequent (5.5\% of all NSCLC cases), but they are quite frequent in East Asian patients (33.4\% of all NSCLC cases) (11). In total 250 different types of mutation have been described, of which a single point mutation in exon 21 (L858R) and a group of small in-frame deletions in exon 19 are the most frequent and account for $\sim 90 \%$ of all EGFR mutations. In addition, the point mutations of the exon 18 in the amino acid residue 719, the L861Q mutation in exon 21 and the in-frame insertions in exon 19 account for $\sim 6 \%$ of all EGFR mutations (12).

Currently, patients with any type of activating EGFR mutation are treated with EGFR-tyrosine kinase inhibitors (TKIs). The reversible EGFR TKIs erlotinib and gefitinib offer a therapeutic alternative for patients with metastatic EGFR mutation-positive lung cancer and are superior to standard 
platinum-based chemotherapy (13). However, the therapeutic success of these targeted agents is limited by primary or acquired drug resistance. Acquired resistance to EGFR-TKIs is either caused by alterations in the EGFR gene that are mediated by a number of molecular mechanisms or by an EGFR-independent mechanism that is facilitated by the activation of alternative signaling pathways to support tumor growth. The T790M mutation is the most frequent mechanism of acquired resistance to EGFR-TKIs in NSCLC $(14,15)$. To date, studies have demonstrated that $50-60 \%$ of tumors have acquired resistance to EGFR-TKIs $(10,16)$. The mono-anilino-pyrimidine compound AZD9291, as a representative of third-generation inhibitors, is a novel, irreversible EGFR-TKI that has been demonstrated to be effective against T790M resistance and EGFR-TKI sensitizing mutations in preclinical models (17). However, resistance to third-generation inhibitors develops rapidly and the EGFR C797S mutation is the main mechanism (18). Therefore, in order to overcome acquired resistance to the third-generation inhibitors, next-generation EGFR-TKIs may be an ideal therapeutic solution.

Currently, genetic alterations in EGFR can be evaluated with a variety of technologies. In general, sequencing, albeit with the limitation of low sensitivity, is the most common method capable of screening all the mutations and includes pyrosequencing, high resolution melting analysis (HRMA) and single-strand conformation polymorphism analysis (SSCP). However, direct sequencing and pyrosequencing can directly identify the mutation, while HRMA and SSCP can only identify the absence or presence of a mutation (19). Therefore, a method to identify the mutations is crucial because of the effect on the therapeutic choice. Quantitative polymerase chain reaction (PCR) is the foundation of most of the methods that detect specific mutations, including amplification refractory mutation system (ARMS) technology $(20,21)$. The source of biological material is one of the most important issues for these molecular tests. In general, sampling the tumor tissue is considered to be the gold standard for genotyping. However, tumor tissue is unavailable in most cases as most patients have tumor metastasis or undergo resection upon diagnosis. Therefore, circulating tumor (ct) DNA is emerging as a novel strategy for genotyping. ctDNA can provide the same information as tumor tissue and avoids tumor heterogeneity, so it can be used in cases where tumor tissue is unavailable (22). At present, various methods are used to detect EGFR mutations in the plasma or serum of patients with NSCLC. Among them, droplet digital PCR (ddPCR) is a novel method that can quantify absolute nucleic acids without using calibration curves (23). This method depends on the ultimate dilutions of the PCR mix volume and Poisson statistics (24-26). Following the ultimate dilutions, the PCR mix is distributed into multiple reactions and the copy number of the target nucleic acids in each reaction is independently tested with single-molecule sensitivity (27). Poisson statistics can be used to calculate the absolute copy number of the target nucleic acids in the original sample $(28,29)$. Compared with traditional PCR and qPCR, the major advantages of ddPCR were to provide a high sensitivity and an absolute quantification for detection. In addition, the development of ddPCR assays for detection of EGFR mutations with high specificity has been reported by a large number of studies (30-32).
In the present study, highly sensitive and specific ddPCR assays were developed for detecting four mutations of the EGFR gene. The sensitivity reached $0.04 \%$. A total of 50 plasma samples were tested by the assays and ARMS-PCR. The ddPCR assays could detect the mutations in the plasma earlier than ARMS-PCR. The consistency was between the ddPCR assays and ARMS-PCR was up to $96 \%$.

\section{Materials and methods}

Cells, samples and reagent kits. The human NSCLC cell line H1975 with mutations of L858R and T790M in EGFR, the human NSCLC cell line H1650 and the cervical carcinoma cell line Hela with mutation of 19DELs, and the wild-type colon carcinoma Caco-2 cell line were obtained from the Institute of Cell Sciences, Shanghai Institutes for Biological Sciences, Chinese Academy of Sciences (Shanghai, China). Peripheral blood samples and paraffin-embedded tissue samples from advanced lung cancer patients were obtained from the Third Affiliated Hospital of Sun Yat-sen University (Guangzhou, China). Acquisition of the samples was approved by the Research Ethics Committee of the Third Affiliated Hospital of Sun Yat-sen University and all subjects provided informed consent.

All synthetic oligonucleotides were purchased from Sangon Biotech Co., Ltd. (Shanghai, China). The peptide nucleic acid (PNA) was synthesized by Panagene (Daejeon, South Korea). Ardent cell-free DNA blood collection tubes were purchased from Ardent BioMed (Mountain view, CA, USA). The droplet reader oil, droplet generation oil for probes, droplet generator cartridges and gaskets, droplet PCR supermix and ddPCR 96-well plates were purchased from Bio-Rad Laboratories, Inc. (Hercules, CA, USA).

Cell culture. The Hela and Caco-2 cells were cultured in Dulbecco's modified Eagles medium (Hyclone; GE Healthcare Life Sciences, Logan, UT, USA), while the H1975 cells and H1650 cells were grownin RPMI-1640 medium(Sigma-Aldrich; Merck KGaA, Darmstadt, Germany). The media was supplemented with $10 \%$ fetal bovine serum (Sigma-Aldrich; Merck $\mathrm{KGaA}$ ) and $100 \mathrm{U} / \mathrm{ml}$ penicillin-streptomycin (Sigma-Aldrich; Merck $\mathrm{KGaA}$ ) in a humidified atmosphere of $5 \% \mathrm{CO}_{2}$ at $37^{\circ} \mathrm{C}$.

Primer design. The primers and specific Minor Groove Binder (MGB) probes for the L858R, T790M and C797S mutations in EGFR were designed using Oligo 7.37 software (Molecular Biology Insights, Inc., Colorado Springs, CO, USA). The sequences of the primers and probes were used in ddPCR and ARMS-PCR as stated in Table I.

Clinical sample processing and DNA extraction. In order to extract the ctDNA in the plasma from the clinical samples, $5 \mathrm{ml}$ blood samples were collected in tubes and centrifuged at $16,000 \mathrm{xg}$ for $10 \mathrm{~min}$ at $4^{\circ} \mathrm{C}$ to obtain the plasma. The ctDNA in the plasma was then extracted using a QIAamp Circulating Nucleic Acid kit (Qiagen GmbH, Hilden, Germany) according to the manufacturer's protocol. In order to obtain the genomic DNA from the appropriate clinical samples of paraffin-embedded tissue, a total of $350 \mu 1$ xylene was added to the samples and then they were centrifuged at $13,000 \mathrm{x} \mathrm{g}$ 
Table I. Sequence of primers and probes.

A, Droplet digital-PCR

\begin{tabular}{ll}
\hline Primer & \multicolumn{1}{c}{ Sequence (5'-3') } \\
\hline ET7-F & CTCCAGGAAGCCTACGTGA \\
ET7-R & GACATAGTCCAGGAGGCAGC \\
ET7-MP & FAM-CAGCTCATCATGCAGCTC-MGB \\
ET7-WP & VIC-CAGCTCATCACGCAGCT-MGB \\
EL8-F & TGGCAGCCAGGAACGTACT \\
EL8-R & TGCCTCCTTCTGCATGGTAT \\
EL8-MP & FAM-TTTGGGCGGGCCAAACT-MGB \\
EL8-WP & VIC-TTTGGGCTGGCCAAACT-MGB \\
E19D-F & TCTCTGTCATAGGGACTCTGG \\
E19D-R & GAGGATTTCCTTGTTGGCT \\
E19D-P & FAM-AAAGCCAACAAGGAAATCC-MGB \\
E19D-B & TGCTTCTCTTAATTC \\
E19D-CF & CTCAGCCTCCAGAGGATGTT \\
E19D-CR & GGAAAATCAAAGTCACCAACC \\
E19D-CP & VIC-AGGTGGTCCTTGGGAATTTG-MGB \\
EC-F & CATCTGCCTCACCTCCACC \\
EC-R & CTTTGTGTTCCCGGACATAGT \\
EC-MP1 & FAM-CTTCGGCAGCCTCCT-MGB \\
EC-MP2 & FAM-CTTCGGCTCCCTCCTG-MGB \\
EC-WP1 & VIC-CTTCGGCTGCCTCCT-MGB \\
\end{tabular}

$\mathrm{B}$, Amplification refractory mutation system-PCR

\begin{tabular}{ll} 
Primer & \multicolumn{1}{c}{ Sequence (5'-3') } \\
\hline T7-F & CACCGTGCAGCTCATCGT \\
T7-R & CCTTCCCTGATTACCTTTGC \\
T7-P & FAM-CCTTCGGCTGCCTCCTG-MGB \\
L8-F & CAAGATCACAGATTTTGGGAG \\
L8-R & CCTGGTCCCTGGTGTCA \\
L8-P & FAM-CAAACTGCTGGGTGCGGA-MGB \\
19D-F1 & TCCCGTCGCTATCAAAACA \\
19D-F2 & CCGTCGCTATCAAGGAATC \\
19D-F3 & CCCGTCGCTATCAAGGC \\
19D-F4 & TCGTCGCTATCAAGGATC \\
19D-F5 & CGTCGCTATCAAGGCATC \\
19D-R & GGATGTGGAGATGAGCAGG \\
19D-P & FAM-AAATCCTCGATGTGAGTTTCTG \\
& C-MGB \\
C-F1 & TCATGCCCTTCGGCAC \\
C-F2 & GCTCATGCCCTTCGGAA \\
C-R & GCAGACCGCATGTGAGGAT \\
C-P & FAM-ATTGGCTCCCAGTACCTGCTC-MGB \\
GAPDH-F & ACAACAGCCTCAAGATCATCAG \\
GAPDH-R & GGTGCTAAGCAGTTGGTGG \\
GAPDH-P & FAM-CCTGGCACCCTATGGACACGC-MGB \\
\hline
\end{tabular}

$\mathrm{MP}$, mutant probe; WP, wild-type probe; $-\mathrm{F}$ or $-\mathrm{CF}$, forward primer; $-\mathrm{R}$ or $-\mathrm{CR}$, reverse primer; $-\mathrm{P}$ or $-\mathrm{CP}$, probe; $-\mathrm{B}$, blocking primer.

for $2 \mathrm{~min}$ at room temperature. Subsequently, $350 \mu \mathrm{l}$ alcohol was added to the sediment and it was drained with a vacuum pump for $\sim 0.5-1 \mathrm{~h}$. The genomic DNA was separated from the cells using a DNA extraction kit (DAAN Gene Co., Ltd., of Sun Yat-sen University, Guangzhou, China) according to the manufacturer's protocol.

To evaluate the DNA copy numbers of L858R, T790M and 19DELs, the DNA from the H1975 cells (harboring the L858R and T790M mutations) and Hela cells (harboring the 19DELs mutation) was extracted and diluted to $50 \mathrm{ng} / \mu \mathrm{l}$. The plasmids of L858R, T790M, 19DELs, C797S and the wild-types were dissolved and diluted to $10^{-6}$ using sterile double distilled water.

Optimization of digital PCR amplification. PCR reactions were assembled into the individual wells of a single-use injection molded cartridge. Subsequently, $70 \mu \mathrm{l}$ droplet generation oil was loaded and the cartridge was placed into the droplet generator. Mono-dispersed droplets were generated by mixing the sample and oil and $40 \mu \mathrm{l}$ were transferred into a 96-well PCR plate for the thermal cycling amplification. The reaction conditions for the L858R, T790M and C797S mutations were as follows: $95^{\circ} \mathrm{C}$ for $10 \mathrm{~min} ; 94^{\circ} \mathrm{C}$ for $30 \mathrm{sec} ; 39$ cycles of $60^{\circ} \mathrm{C}(\mathrm{C} 797 \mathrm{~S}), 58^{\circ} \mathrm{C}(\mathrm{L} 858 \mathrm{R})$ or $54^{\circ} \mathrm{C}(\mathrm{T} 790 \mathrm{M})$ for $60 \mathrm{sec}$; $98^{\circ} \mathrm{C}$ for $10 \mathrm{~min}$; and $4^{\circ} \mathrm{C}$ hold. The reaction conditions for the 19DELs mutations were as follows: $95^{\circ} \mathrm{C}$ for $10 \mathrm{~min} ; 94^{\circ} \mathrm{C}$ for $30 \mathrm{sec} ; 70^{\circ} \mathrm{C}$ for $10 \mathrm{sec} ; 39$ cycles of $60^{\circ} \mathrm{C}$ for $60 \mathrm{sec} ; 98^{\circ} \mathrm{C}$ for $10 \mathrm{~min}$; and $4^{\circ} \mathrm{C}$ hold. A droplet reader (Bio-Rad Laboratories, Inc.) was used for fluorescence signal quantification. A total of four negative controls were routinely included to reduce false-positive results. Human internal reference genomic DNA (GAPDH) was routinely used as a negative control to determine the cut-off for allele calling. The PCR reactions of L858R, T790M, C797S and 19DELs are presented in Table II.

Evaluation of DNA copy number and sensitivity testing of the ddPCR assays. After the PCR amplification reactions, the droplet reader was used for fluorescence signal quantification. According to the copy number of DNA, the extracted DNA and plasmid of C797S was diluted to 3,000 copies $/ \mu 1$. To evaluate the sensitivity of the L858R, T790M and 19DELs assays, the DNA solutions from the H1975 cells (harboring the L858R and T790M mutations), Hela cells (harboring the 19DELs mutation) and plasmid were serially diluted with the DNA solution from the Caco-2 cells (wild-type) to achieve decreasing ratios $(1: 10$ to $4: 10,000)$. A total of four negative controls were routinely included to reduce false-positive results. Human internal reference genomic DNA was routinely used as a negative control to determine the cut-off for allele calling.

EGFR mutation detection of plasma ctDNA and paraffin-embedded tumor tissue sample DNA byddPCR assays and ARMS-PCR. The plasma ctDNA and paraffin-embedded tumor tissues samples were tested using the optimal reaction system and amplification conditions of the aforementioned ddPCR assays. The plasma ctDNA and paraffin-embedded tumor tissue samples were also tested using ARMS quantitative PCR. The ARMS quantitative $\mathrm{PCR}$ reaction mixture contained $10 \mathrm{mM}$ Tris- $\mathrm{HCl}$ (pH 8.3), $20 \mathrm{mM} \mathrm{KCl,} 200 \mathrm{mM}$ dNTPs, $3.5 \mathrm{mM} \mathrm{MgCl}$, forward primers (10 pM each), MGB block primers ( $4 \mathrm{pM}$ each), reverse primers (10 pM each), probes (3 pM each) and $\geq 10 \mathrm{ng}$ genomic DNA. PCR amplification 
Table II. PCR reactions system of mutant sites.

\begin{tabular}{llr}
\hline PCR reaction & \multicolumn{1}{c}{ Reagents } & Dosage $(\mu t)$ \\
\hline Mutant PCR reactions of L858R, & 2X Droplet PCR Supermix & 10 \\
T790M and C797S & Mutant primers/FAM probe $(450 \mathrm{nM} / 250 \mathrm{nM})$ & 1 \\
& Sterile double distilled water & 5 \\
Wild-type PCR reactions of & Mutant/wild-type plasmid & 4 \\
L858R, T790M and C797S & 2X Droplet PCR Supermix & 10 \\
& Wild-type primers/VIC probe $(450 \mathrm{nM} / 250 \mathrm{nM})$ & 1 \\
& Sterile double distilled water & 5 \\
Mutant PCR reaction of 19DELs & Mutant/wild-type plasmid & 4 \\
& 2X Droplet PCR Supermix & 10 \\
& Mutant primers/FAM probe $(450 \mathrm{nM} / 250 \mathrm{nM})$ & 1 \\
& PNA (450 nM) & 4
\end{tabular}

PCR, polymerase chain reaction; PNA, peptide nucleic acid.

19DELS

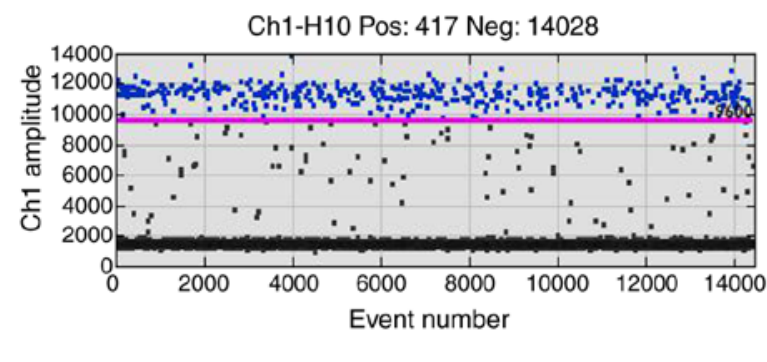

T790M

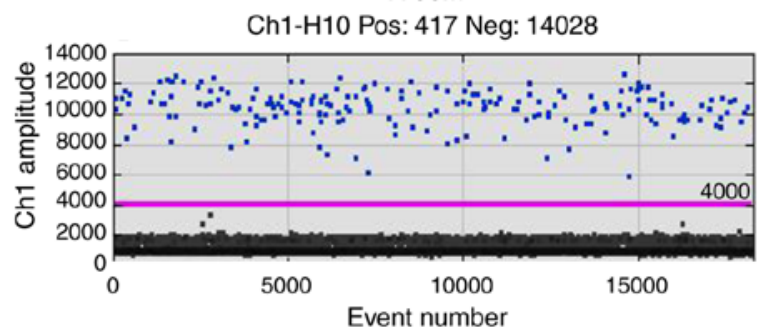

L858R

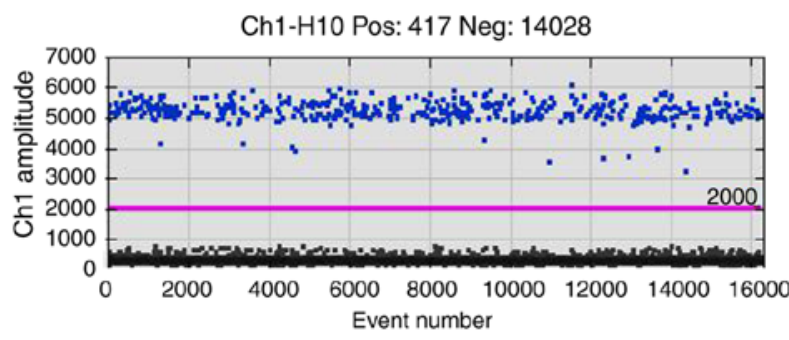

C797S

Ch1-H10 Pos: 417 Neg: 14028

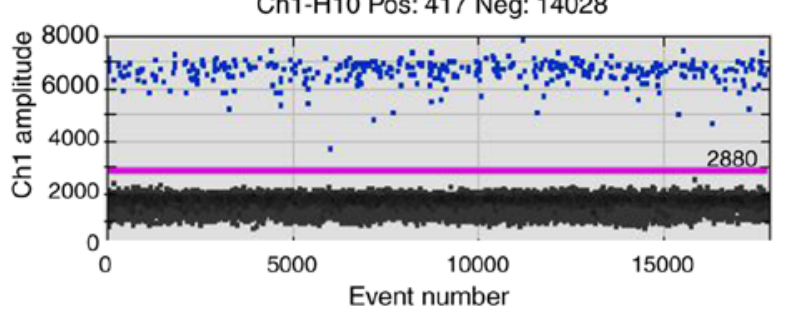

Figure 1. Specificity of the ddPCR for 19DELs, L858R, T790M and C797S mutations. Representation of the specificity results of the ddPCR for 19DELs, L858R, T790M and C797S mutations. ddPCR, droplet digital polymerase chain reaction; Pos, positive; Neg, negative.

was performed using an ABI7500 Real-Time PCR system (Applied Biosystems; Thermo Fisher Scientific, Inc., Waltham, MA, USA) with the following protocol: $50^{\circ} \mathrm{C}$ for $2 \mathrm{~min} ; 95^{\circ} \mathrm{C}$ for $5 \mathrm{~min}$; 40 cycles of $94^{\circ} \mathrm{C}$ for $15 \mathrm{sec}$ and $55^{\circ} \mathrm{C}$ for $45 \mathrm{sec}$. In the ARMS-PCR amplification system, the housekeeping gene GAPDH was used as the internal control gene and the results were directly obtained by the $\mathrm{Cq}$ value (33). When the $\mathrm{Cq}$ value of the tested sample was $\leq 36$, it was considered to be positive and $>36$ was considered to be negative.

Statistical analysis. The ddPCR data for allele calling was analyzed using QuantaSoft software, version 1.7.0 (Bio-Rad Laboratories, Inc.). Analysis of the results of the ARMS-PCR data was performed with SDSShell 2.0 software (Applied Biosystems; Thermo Fisher Scientific, Inc.).

\section{Results}

Specificity of the primers and probes. The specificity for all mutations of EGFR was tested. The wild-type plasmid (containing the wild-type EGFR) exhibited high fluorescence signal in the 2'-chloro-7'phenyl-1,4-dichloro-6-carboxy-fluorescein (VIC) channel and no fluorescence signal in the fluorescein amidite (FAM) channel. However, the mutant plasmid (containing the mutant EGFR) exhibited high fluorescence signal in the FAM channel and no fluorescence signal in the VIC channel, meaning that the primers and probes exhibited good specificity. To develop the detection assays of the EGFR mutations, the MGB probe was introduced to improve the specificity of the assays. MGB can bind in the minor groove of double-stranded DNA as its small size 
Table III. Copy numbers of DNA and plasmid.

\begin{tabular}{lccc}
\hline Template & Copies/4 $\mu 1$ & Copies/ng & $\begin{array}{c}\text { Mutation } \\
\text { ratio (\%) }\end{array}$ \\
\hline H1975 (L858R) & 23,000 & 115 & $78.3 \%$ \\
H1975 (T790M) & 23,000 & 115 & $79.5 \%$ \\
Hela (19DELs) & 8,851 & 44 & $33.4 \%$ \\
Caco-2 & 9,622 & 48 & $/$ \\
Plasmid & 12,736 & $/$ & $/$ \\
\hline
\end{tabular}

Table IV. Mutation ratio of 20 repetitions.

\begin{tabular}{llll}
\hline 19DELs (\%) & L858R $(\%)$ & T790M $(\%)$ & C797S $(\%)$ \\
\hline 0.028 & 0.026 & 0.08 & 0.031 \\
0.039 & 0.037 & 0.07 & 0.011 \\
0.027 & 0.047 & 0.032 & 0.09 \\
0.028 & 0.043 & 0.013 & 0.031 \\
0.022 & 0.045 & 0.07 & 0.021 \\
0.022 & 0.028 & 0.1 & 0.018 \\
0.06 & 0.045 & 0.11 & 0.08 \\
0.04 & 0.038 & 0.08 & 0.02 \\
0.032 & 0.08 & 0.07 & 0.02 \\
0.048 & 0.028 & 0.06 & 0.035 \\
0.033 & 0.031 & 0.14 & 0.039 \\
0.036 & 0.07 & 0.08 & 0.056 \\
0.054 & 0.012 & 0.75 & 0.025 \\
0.07 & 0.06 & 0.056 & 0.011 \\
0.06 & 0.013 & 0.076 & 0.042 \\
0.05 & 0.05 & 0.066 & 0.08 \\
0.011 & 0.013 & 0.022 & 0.021 \\
0.011 & 0.044 & 0.08 & 0.053 \\
0.017 & 0.024 & 0.05 & 0.024 \\
0.021 & 0.027 & 0.038 & 0.05 \\
\hline
\end{tabular}

leads to a high melting temperature $(22,23)$. According to the results, the mutation amplification reactions of the four assays were of high specificity, which suggested that the MGB probe can distinguish a single mismatch between the wild-type and mutation-type (Fig. 1). According to the fluorescent signal of the negative control, the threshold values of 19DELs, L858R, T790M and C797S were 9,600, 2,000, 4,000 and 2,880, respectively. The 19DELs reactions exhibited higher threshold values compared with the other three reactions due to the use of PNA to block amplification of the wild-type (Fig. 1). There were large micro-reactions with different fluorescence signals in the FAM channel, which may be due to the different blocking efficiency of PNA in micro-reactions.

Sensitivity of the assays. In order to evaluate the sensitivity of the assay for EGFR mutation, the copy number of $50 \mathrm{ng} / \mu \mathrm{l}$ DNA from cells and plasmids was tested. It is known that
33 ng of human genomic DNA contains 10,000 copies. The total copy numbers of the three cell types were different and described in Table III. Through testing serial dilutions of EGFR mutations by mixing mutation-type DNA derived from the positive cell lines (H1650 and Hela for 19DELs, and H1975 for L858R and T790M) with wild-type DNA from the Caco-2 cell line, it was possible to stably detect the four major types of EGFR mutation until the ratio of mutation-type to wild-type was 1:2,500 (0.04\%; Figs. 2 and 3). Although the mutation ratios were different in the 20 repetitions, all the reactions were mutation-positive (Table IV). Therefore, the selective sensitivity of the ddPCR assays for 19DELs, L858R, T790M and C797S was $0.04 \%$ in the testing of cell line DNA (Figs. 2 and 3). Digital PCR is the third generation of PCR with a high sensitivity, which can reach $0.001-0.0001 \%$ (25). In the present study, although the sensitivity of the ddPCR assays reached $0.04 \%$, it is lower than the theoretical amount; therefore, further optimization of the assays to obtain a more sensitive result is required.

EGFR mutation testing by ddPCR and ARMS-PCR in clinical plasma ctDNA samples. In total, 50 plasma ctDNA samples collected from patients with EGFR TKI-naive NSCLC were tested by ddPCR and ARMS-PCR for the mutations of EGFR exon19 deletions, L858R, T790M and C797S. All 50 samples were negative for $\mathrm{C} 797 \mathrm{~S}$, which may be due to the rarity of the C797S mutation (34). For the remaining mutation types, 11 plasma samples were positive for the 19DELs in the ddPCR assay, while 9 plasma samples of the 11 were also positive for 19DELs by ARMS-PCR (Fig. 4). Therefore, the concordance rate between ddPCR and ARMS-PCR was 96\% (48 of 50) in the 19DELs mutation testing (Fig. 5). In the L858R ddPCR assay, 7 plasma samples were identified to be positive, but 6 plasma samples of the 7 were also positive for L858R by ARMS-PCR (Fig. 4). Therefore, the concordance rate between ddPCR and ARMS-PCR was 98\% (49 of 50) in the L858R mutation testing (Fig. 5). A total of 5 plasma samples were identified to be positive in the T790M ddPCR assay as well as the ARMS-PCR testing. Therefore, the concordance rate between ddPCR and ARMS-PCR was 100\% (50 of 50) in the T790M mutation testing (Fig. 5). In conclusion, for these 23 samples, the fraction with each EGFR mutation ranged from 0.43 to $68.07 \%$ and three plasma samples were positive for EGFR mutations by ddPCR but negative by ARMS-PCR testing. The EGFR mutation fractions of these three plasma samples were $0.43,0.67$ and $0.86 \%$, respectively (Table V), which were generally low compared to the other positive plasma samples but still much higher than the $0.04 \%$ detection limit of the ddPCR assays. The results suggested that the ddPCR assays exhibit higher sensitivity compared to the ARMS-PCR assays.

EGFR mutation testing by ddPCR and ARMS-PCR in paraffin-embedded tumor tissue samples. In the plasma testing, three samples were positive for EGFR mutations by ddPCR but were negative by ARMS-PCR testing, as they had extremely low fractions of mutation DNA. However, the possibility of experimental artifacts could not be completely ruled out. Therefore, the EGFR mutation was also detected by ddPCR and ARMS-PCR in the paraffin-embedded tumor tissues samples of the three patients. The results revealed that 

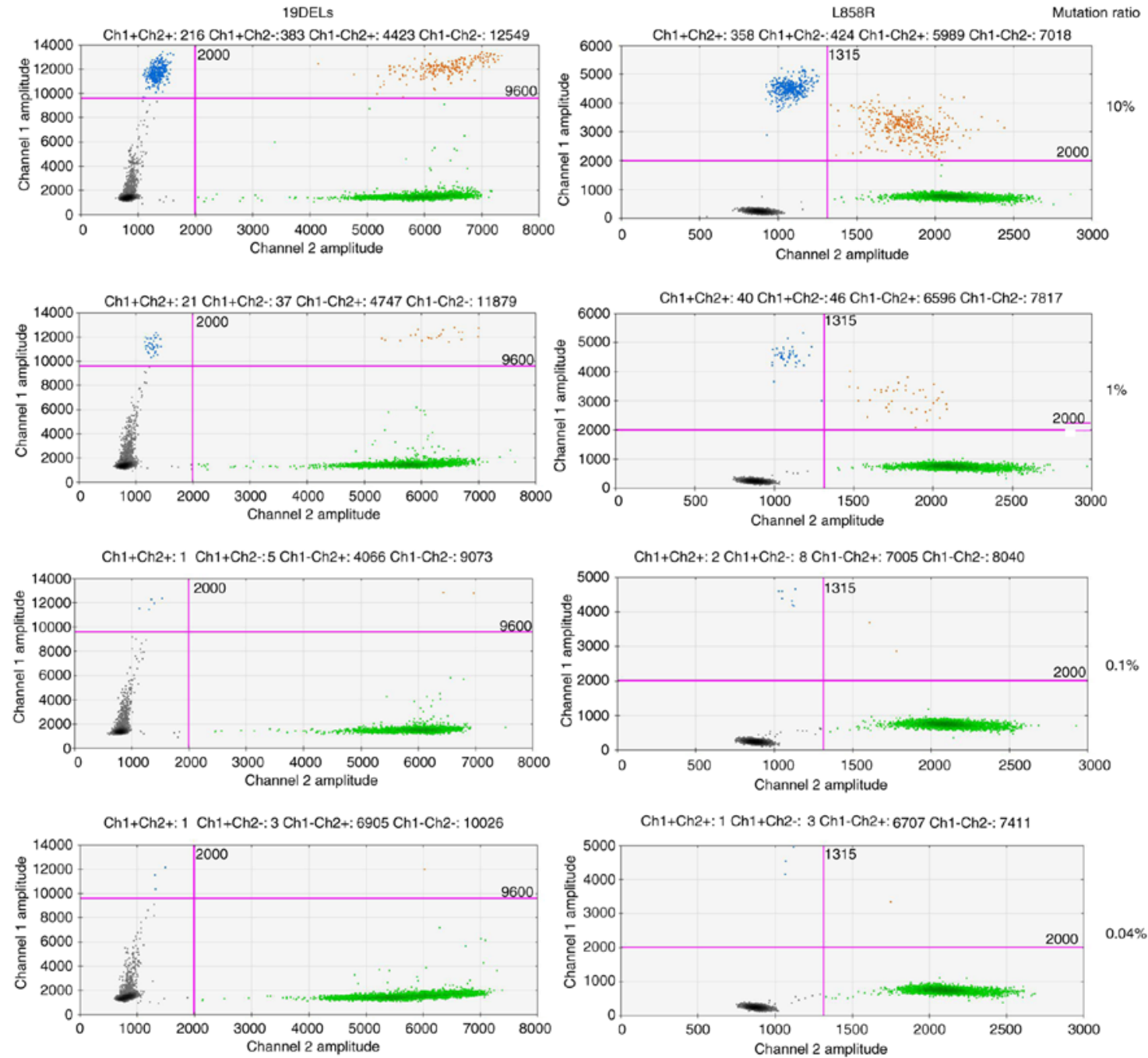

Figure 2. Sensitivity of the ddPCR for EGFR 19DELs and L858R mutations. Up to 0.04\% dilution of mutation-type to wild-type of EGFR, at least two positive droplets were stably detected by ddPCR assays. The numbers presented in the positive area were the amounts of EGFR mutation positive droplets by detection ddPCR, droplet digital polymerase chain reaction; EGFR, epidermal growth factor receptor.

Table V. Summary of EGFR mutation fraction of $23 E G F R$ mutation-positive plasma samples.

\begin{tabular}{lccc}
\hline $\begin{array}{l}\text { Plasma } \\
\text { samples }\end{array}$ & $\begin{array}{c}\text { Summary of } \\
\text { EGFR mutation } \\
\text { fraction (\%) }\end{array}$ & $\begin{array}{c}\text { Plasma } \\
\text { samples }\end{array}$ & $\begin{array}{c}\text { Summary of } \\
\text { EGFR mutation } \\
\text { fraction (\%) }\end{array}$ \\
\hline 1 & 22.48 & 13 & 32.76 \\
2 & 15.76 & 14 & 28.96 \\
3 & 5.67 & 15 & 0.43 \\
4 & 30.56 & 16 & 26.67 \\
5 & 8.91 & 17 & 0.67 \\
6 & 14.48 & 18 & 13.48 \\
7 & 0.86 & 19 & 12.56 \\
8 & 16.75 & 20 & 3.59 \\
9 & 15.62 & 21 & 11.78 \\
10 & 68.07 & 22 & 26.86 \\
11 & 23.46 & 23 & 19.48 \\
12 & 44.76 & & \\
\hline
\end{tabular}

EGFR, epidermal growth factor receptor. two paraffin-embedded tumor tissues samples were identified to be 19DELs mutation-positive and one paraffin-embedded tumor tissues sample was identified to be L858R mutation-positive by ddPCR, which was consistent with the plasma samples (Figs. 4 and 6). Therefore, the result of the previous small mutation detection by digital PCR was correct. This suggested that the ddPCR assays exhibited higher sensitivity compared to ARMS-PCR assays.

\section{Discussion}

Lung cancer is one of the most common types of malignant tumor worldwide and NSCLC is the leading cause of cancer-associated mortalities globally (35). Molecular studies have demonstrated that EGFR is a key factor in NSCLC tumor development (5). Tissue biopsy is the current standard diagnostic procedure and it has high reliability, but the success rate is poor. A previous study revealed that $\sim 10-50 \%$ of patients undergo a biopsy but not enough tumor tissue is obtained for EGFR genotyping (36). Therefore, a non-invasive, quantitative, blood-based ctDNA assay is urgently required as a novel strategy for the detection of 
T790M
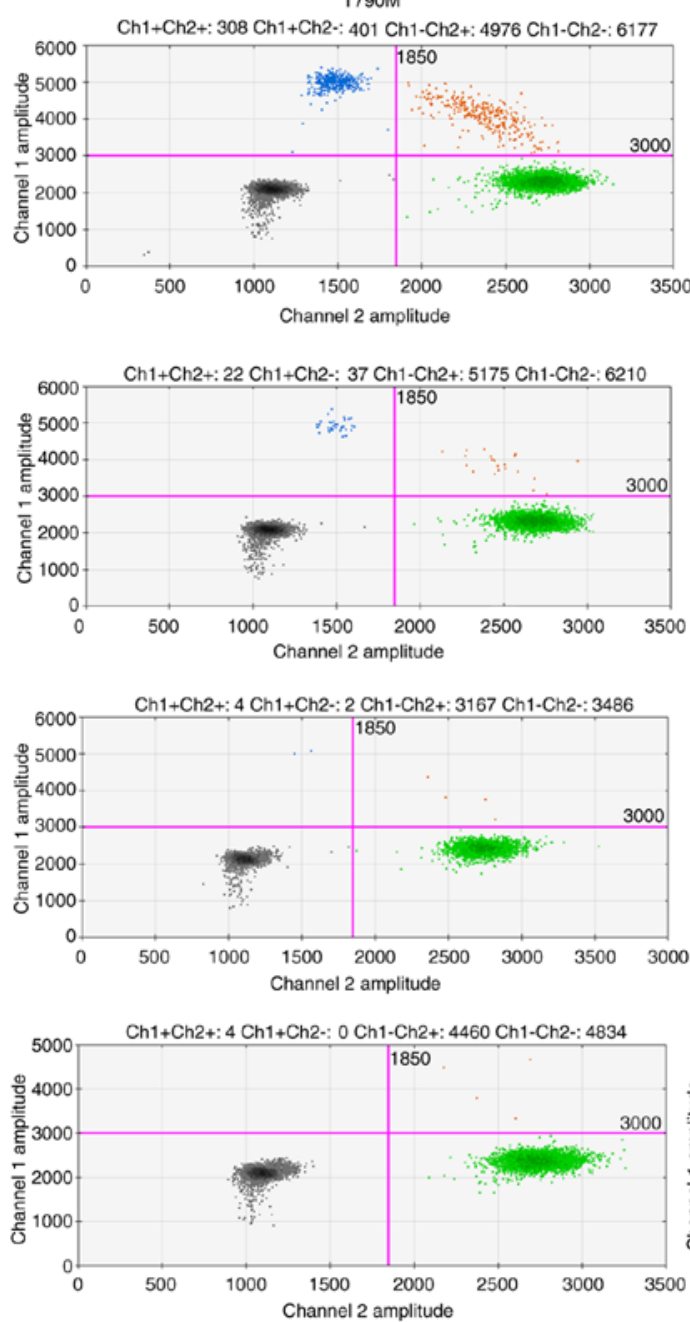

C797S
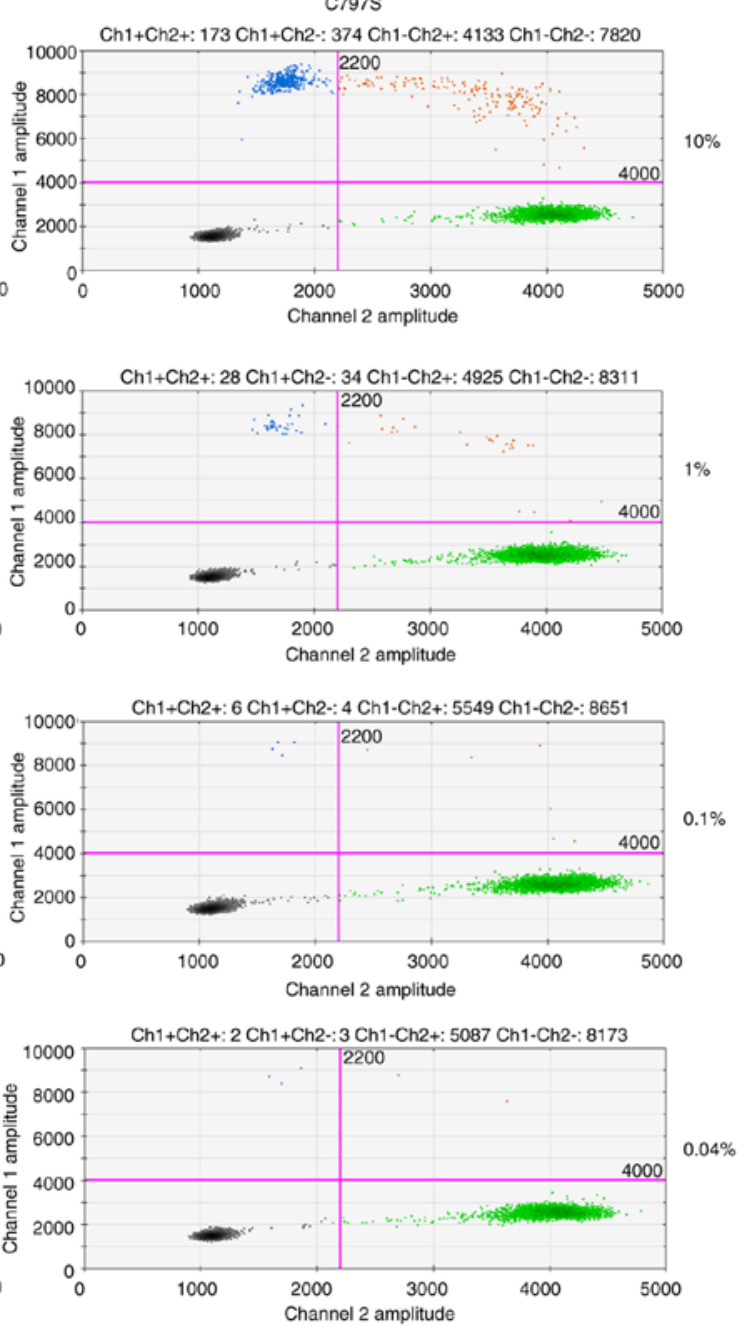

Figure 3. Sensitivity of the ddPCR for EGFR T790M and C797S mutations. Up to 0.04\% dilution of mutation-type to wild-type of EGFR, at least two positive droplets were stably detected by ddPCR assays. The numbers presented in the positive area were the amounts of EGFR mutation positive droplets by detection. ddPCR, droplet digital polymerase chain reaction; EGFR, epidermal growth factor receptor.

EGFR-activating mutations. However, for EGFR mutations, the analysis of plasma ctDNA has technical challenges. For example, the tumor-derived ctDNA fraction is low in quantity and variable between individuals. Therefore, in the present study, four ddPCR testing systems for 19DELs, L858R, T790M and C797S were developed to detect these EGFR mutations with high sensitivity.

There are numerous methods to detect EGFR gene mutation (15-18); however, they vary widely in sensitivity, assay complexity and cost. For example, automated dideoxy sequencing has been considered the gold standard for mutation detection, but its sensitivity only reaches $10-20 \%$. Allele-specific PCR is a huge improvement with $1-5 \%$ sensitivity. Researchers are focusing on developing more sensitive and easy-to-use methods (37). ddPCR is emerging as a method to detect a low abundance of plasma ctDNA with ultra-sensitivity and absolute quantification. Digital PCR is the third generation of PCR and has high sensitivity, which can reach 0.001-0.0001\%. In the present study, a novel digital PCR method was developed to detect EGFR mutations, which had high sensitivity in plasma ctDNA. Furthermore, a 15-bp PNA was introduced to block amplification of the wild-type EGFR allele with high efficiency. It was possible to detect the four major types of EGFR mutation and the selective sensitivity of the assays achieved was at least $0.04 \%$ in 20 stable repetitions. In addition, highly sensitive ddPCR may avoid false negative results that are associated with tumor heterogeneity in the evaluation of enriched samples in tumor cells (23).

In the present study, 50 plasma samples of patients with NSCLC were tested using ddPCR assays and ARMS-PCR. A total of three mutations were detected, which were 19DELs, T790M and L861Q, and the concordance rate between ddPCR and ARMS-PCR was 96, 98 and 100\%, respectively. For the 23 positive samples, the fraction of EGFR mutation ranged from 0.43 to $68.07 \%$ and three plasma samples were positive for EGFR mutations by ddPCR but negative by ARMS-PCR testing. However, the positive mutation rate of these three plasma samples was $0.43,0.67$ and $0.86 \%$, respectively (Table V), which may not be detected by ARMS-PCR. Therefore, in order to verify the results, paraffin-embedded tumor tissues samples from these three patients were collected and simultaneously detected by digital PCR and ARMS-PCR. The results revealed that two of the three paraffin-embedded 
ddPCR

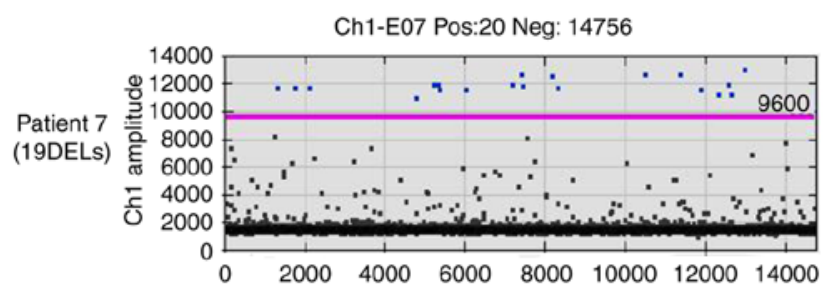

Event number

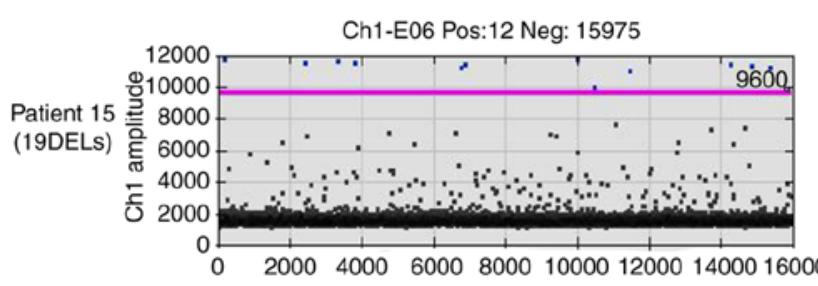

Event number

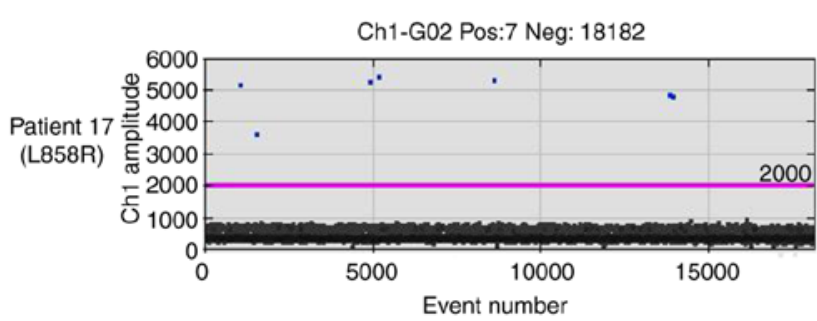

ARMS-PCR

Amplification plot

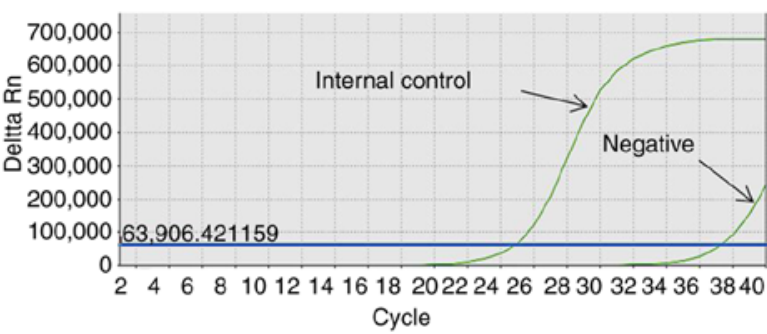

Amplification plot

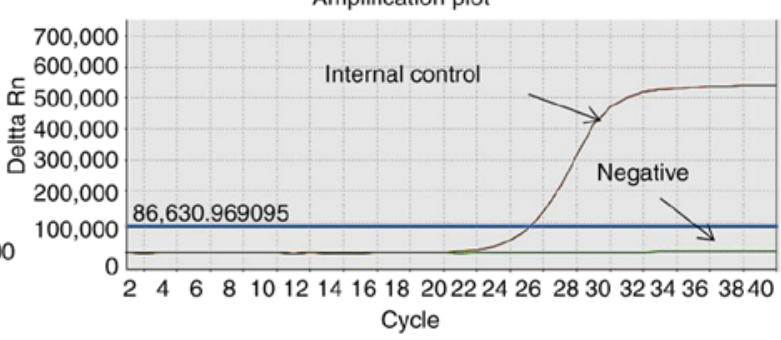

Amplification plot

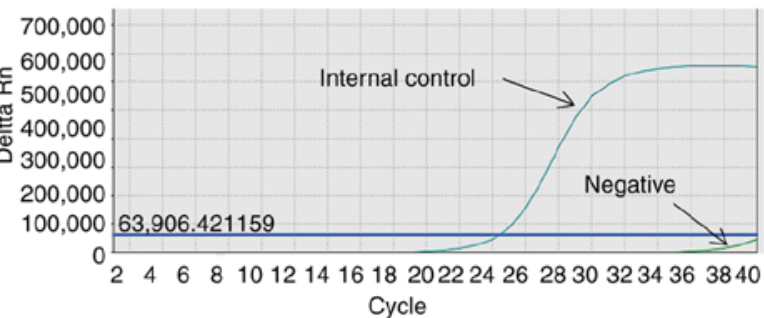

Figure 4. EGFR mutation testing by ddPCR and ARMS-PCR in clinical plasma ctDNA samples. A total of 50 plasma ctDNA samples collected from EGFR tyrosine kinase inhibitor-naive non-small cell lung cancer patients were tested respectively by ddPCR and ARMs-PCR for the mutations of EGFR 19DELs, L858R, T790M. The testing results by ddPCR and ARMs-PCR of patient 7, 15 and 17 are illustrated. ddPCR, droplet digital polymerase chain reaction; EGFR, epidermal growth factor receptor; ARMS, amplification refractory mutation system; ct, circulating tumor; Pos, positive; Neg, negative.

19DELs

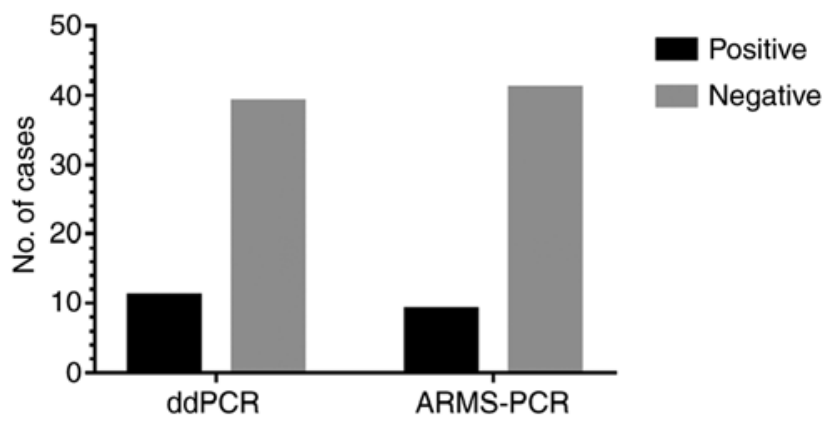

L858R

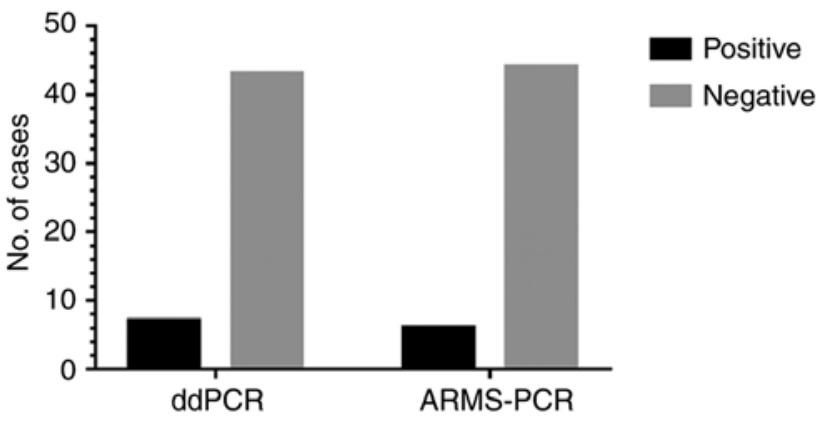

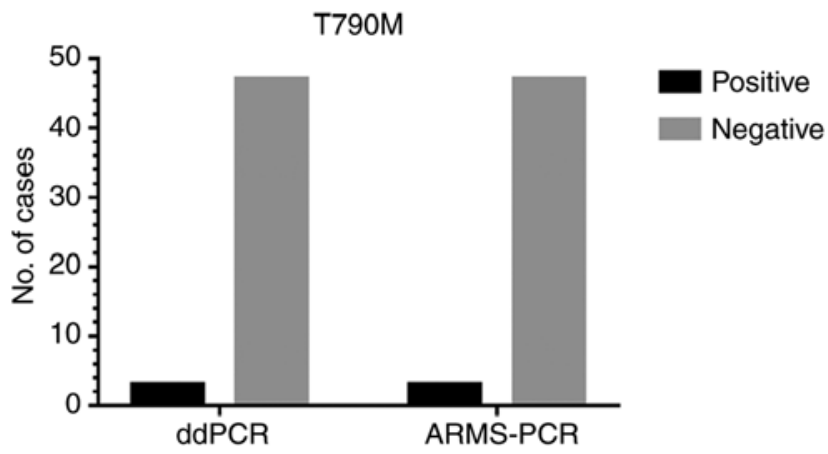

Figure 5. Comparison of EGFR 19DELs, L858R and T790M detected by ddPCR and ARMS-PCR in plasma circulating tumor DNA. In the present study there was a total of 50 cases. ddPCR, droplet digital polymerase chain reaction; EGFR, epidermal growth factor receptor; ARMS-PCR, amplification refractory mutation system polymerase chain reaction. 

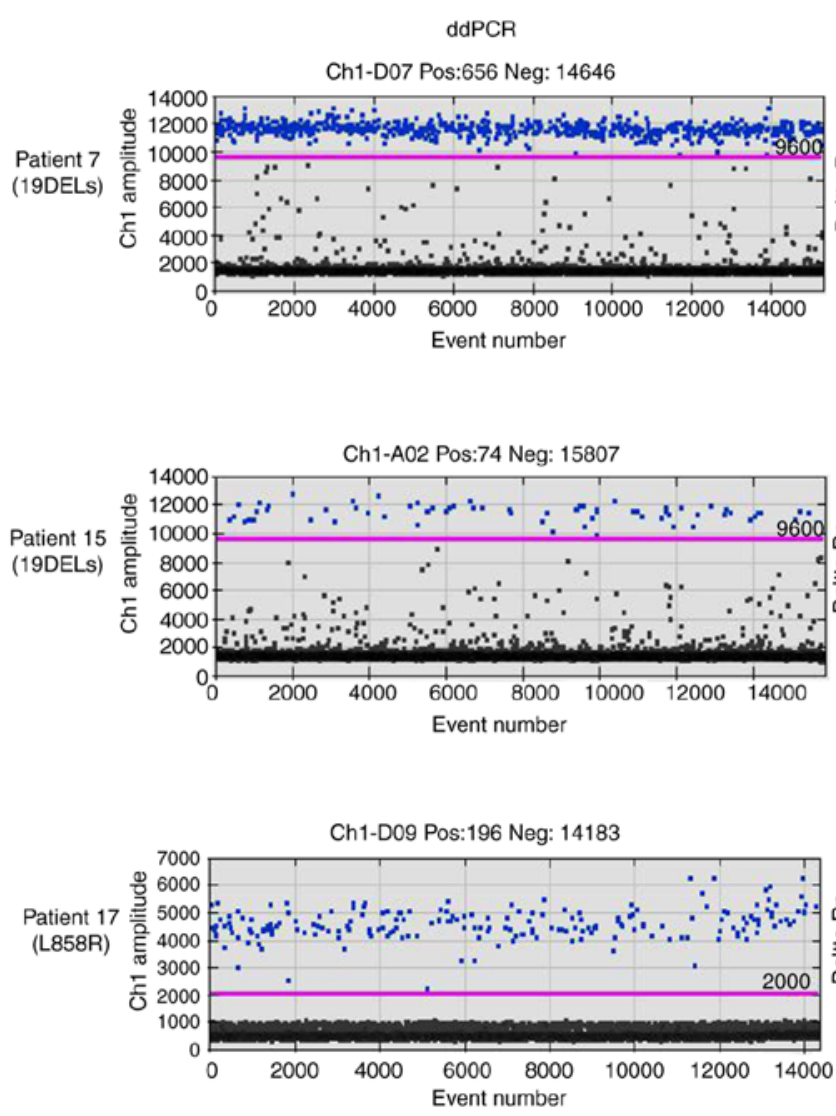

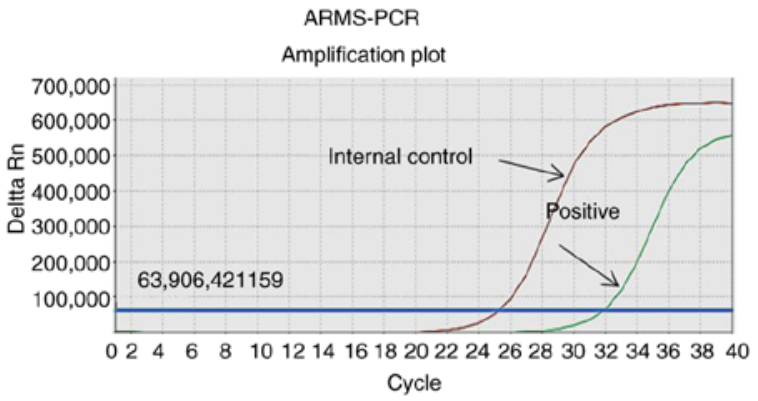

Amplification plot

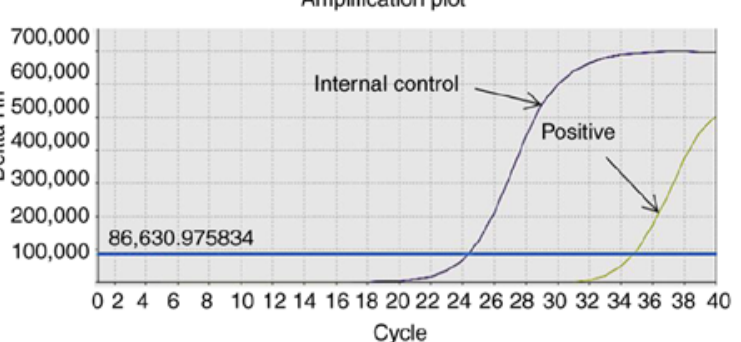

Amplification plot

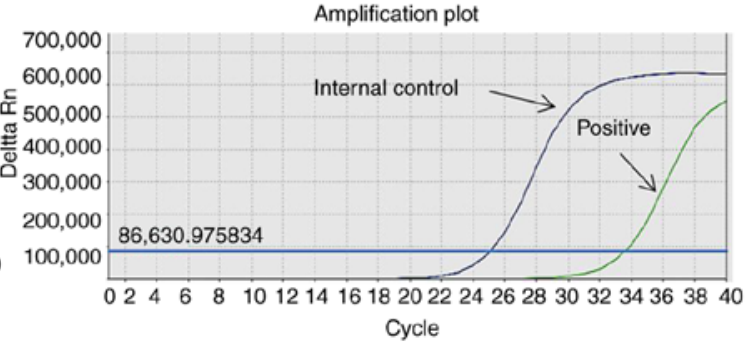

Figure 6. EGFR mutation testing by ddPCR and ARMS-PCR in paraffin tumor tissues samples. The three patients whose EGFR mutations were positive by ddPCR but negative by ARMS-PCR in plasma samples, the paraffin tumor tissues samples also were tested respectively by ddPCR and ARMS-PCR for the mutations of EGFR 19DELs and L858R. The testing results by ddPCR and ARMS-PCR of patient 7, 15 and 17 are illustrated. ddPCR, droplet digital polymerase chain reaction; EGFR, epidermal growth factor receptor; ARMS, amplification refractory mutation system; Pos, positive; Neg, negative.

tumor tissues samples were identified to be 19DELs mutation-positive and the other paraffin-embedded tumor tissue sample was identified to be L858R mutation-positive by the two methods. These results suggest that the ddPCR assays exhibited higher sensitivity compared with the ARMS-PCR assays and ddPCR is able to detect EGFR mutations early in plasma samples.

Furthermore, plasma ctDNA samples of two patients not only had 19DELs, but also had a T790M mutation. In addition, one L858R mutation-positive patient was also T790M mutation-positive by ddPCR testing. A previous study has demonstrated that if patients only have the 19DELs or L858R mutation, they could benefit from first-generation EGFR-targeted TKI drugs (28). However, once the patients have a double mutation with 19DELs and T790M or 19DELs and L858R, they will not benefit from first-generation EGFR-targeted TKI drugs and they may benefit from the third-generation EGFR-targeted TKI drug AZD9291 (29). Therefore, the ddPCR technique may be used for early detection of these mutations in plasma samples, so that the patient can select the targeted drugs according to the EGFR mutation. Blood-based ctDNA can provide a homogeneous representation of all tumor DNA and allow continual monitoring for tumor mutation profiles without invasive biopsies, tissue archives and tumor heterogeneity. Overall, the results suggest that the ddPCR assays exhibit higher sensitivity compared to
ARMS-PCR assays and they can detect EGFR mutations early and noninvasively.

Similar experiments have been done in previous studies. For examples, Kim et al (38) used ddPCR to quantify EGFR mutations in paraffin-embedded tissues with having a high sensitivity and Zhu et al (39) used a novel ARMS-based assay to detect the L858R, 19DELs and T790M mutations of EGFR proving that it demonstrated similar detection effect with ddPCR. However, the present experiment was mainly used to detect plasma cell-free DNA as a novel generation of markers for non-invasive detection of tumors and detected the unusual drug-resistant mutation C797S of EGFR whose detection limit reached $0.04 \%$. Therefore, the present study's digital PCR system has a great advantage over previous studies.

In conclusion, four ddPCR testing systems for the detection of the 19DELs, L858R, T790M and C797S mutations of the EGFR gene in plasma ctDNA samples have been developed, which all exhibit higher sensitivity compared to ARMS-PCR assays. Therefore, the four ddPCR testing systems could be used for early detection of EGFR mutations in plasma samples, so that patients can better select the targeted drugs according to the EGFR mutation.

\section{Acknowledgements}

Not applicable. 


\section{Funding}

The present study was supported by the Science and Technology projects of Guangzhou (grant no. 2016DAGZKJ007).

\section{Availability of data and materials}

All data generated or analyzed during this study are included in this published article.

\section{Authors' contributions}

XJ designed and performed the experiments. WL performed the experiments and analyzed the data. XZ and XX analyzed the data, prepared all of the figures and wrote the paper.

\section{Ethics approval and consent to participate}

The present study was approved by the Research Ethical Committee of the Third Affiliated Hospital of Sun Yat-sen University; all subjects gave informed consent.

\section{Patient consent for publication}

Patient information was anonymized and de-identified prior to analysis and all the patients agreed to publication.

\section{Competing interests}

The authors declare that they have no conflict of interest.

\section{References}

1. Chen W, Zheng R, Zeng $\mathrm{H}$ and Zhang S: The incidence and mortality of major cancers in China, 2012. Chin J Cancer 35: 73, 2016.

2. Kumar M, Ernani V and Owonikoko TK: Biomarkers and targeted systemic therapies in advanced non-small cell lung cancer. Mol Aspects Med 45: 55-66, 2015.

3. American Cancer Society. Lung cancer (non-small cell). 2016 : Available from: http://www.cancer.org/acs/groups/cid/documents/webcontent/003115-pdf.pdf. Accessed October 27, 2016.

4. Signorovitch J, Zhou Z, Ryan J, Anhorn R and Chawla A: Budget impact analysis of comprehensive genomic profiling in patients with advanced non-small cell lung cancer. J Med Econ 22: 140-150, 2019.

5. Zhang Y, Wang Q, Han ZG and Shan L: Differences in epidermal growth factor receptor gene mutations and relationship with clinicopathological features in NSCLC between Uygur and Han ethnic groups. Asian Pac J Cancer Prev 14: 2879-2883, 2013.

6. Shih AJ, Telesco SE and Radhakrishnan R: Analysis of somatic mutations in cancer: Molecular mechanisms of activation in the ErbB family of receptor tyrosine kinases. Cancers 3: 1195-1231, 2011.

7. Dawson JP, Berger MB, Lin CC, Schlessinger J, Lemmon MA and Ferguson KM: Epidermal growth factor receptor dimerization and activation require ligand-induced conformational changes in the dimer interface. Mol Cell Biol 25: 7734-7742, 2005.

8. Luo M and Fu LW: Redundant kinase activation and resistance of EGFR-tyrosine kinase inhibitors. Am J Cancer Res 4: 608-628, 2014.

9. Weiss GA, Rossi MR, Khushalani NI, Lo K, Gibbs JF, Bharthuar A, Cowell JK and Iyer R: Evaluation of phosphatidylinositol-3-kinase catalytic subunit (PIK3CA) and epidermal growth factor receptor (EGFR) gene mutations in pancreaticobiliary adenocarcinoma. J Gastroint Oncol 4: 20-29, 2013.

10. Gazdar AF: Activating and resistance mutations of EGFR in non-small-cell lung cancer: Role in clinical response to EGFR tyrosine kinase inhibitors. Oncogene 28 (Suppl 1): S24-S31, 2009.
11. Normanno N, Luca AD, Bianco C, Strizzi L, Mancino M, Maiello MR, Carotenuto A, De Feo G, Caponigro F and Salomon DS: Epidermal growth factor receptor (EGFR) signaling in cancer. Gene 366: 2-16, 2006.

12. Ma C, Wei S and Song Y: T790M and acquired resistance of EGFR TKI: A literature review of clinical reports. J Thorac Dis 3: 10-18, 2011.

13. Köhler J and Schuler M: Afatinib, erlotinib and gefitinib in the first-line therapy of EGFR mutation-positive lung adenocarcinoma: A review. Onkologie 36: 510-518, 2013.

14. Shih JY, Gow CH and Yang PC: EGFR mutation conferring primary resistance to gefitinib in non-small-cell lung cancer. N Engl J Med 353: 207-208, 2005.

15. Pao W, Miller VA, Politi KA, Riely GJ, Somwar R, Zakowski MF, Kris MG and Varmus H: Acquired resistance of lung adenocarcinomas to gefitinib or erlotinib is associated with a second mutation in the EGFR kinase domain. PLoS Med 2: 225-235, 2005.

16. Kosaka T, Yamaki E, Mogi A and Kuwano H: Mechanisms of resistance to EGFR TKIs and development of a new generation of drugs in non-small-cell lung cancer. J Biomed Biotechnol 2011: 165214,2011

17. Jänne PA, Yang JC, Kim DW, Planchard D, Ohe Y, Ramalingam SS, Ahn MJ, Kim SW, Su WC, Horn L, et al: AZD9291 in EGFR inhibitor-resistant non-small-cell lung cancer. N Engl J Med 372: 1689-1699, 2015.

18. Thress KS, Paweletz CP, Felip E, Cho BC, Stetson D, Dougherty B, Lai Z, Markovets A, Vivancos A, Kuang Y, et al: Acquired EGFR C797S mutation mediates resistance to AZD9291 in non-small cell lung cancer harboring EGFR T790M. Nat Med 21: 560-562, 2015.

19. Lee HJ, Xu X, Kim H, Jin Y, Sun P, Kim JE and Chung JH: Comparison of direct sequencing, PNA clamping-real time polymerase chain reaction, and pyrosequencing methods for the detection of EGFR mutations in non-small cell lung carcinoma and the correlation with clinical responses to EGFR tyrosine kinase inhibitor treatment. Korean J Pathol 47: 52-60, 2013.

20. Huang T, Zhuge J and Zhang WW: Sensitive detection of BRAF V600E mutation by amplification refractory mutation system (ARMS)-PCR. Biomark Res 1: 3, 2013.

21. Liu Y,Liu B, Li XY, Li JJ, Qin HF, Tang CH, Guo WF, Hu HX, Li S, Chen CJ, et al: A comparison of ARMS and direct sequencing for EGFR mutation analysis and tyrosine kinase inhibitors treatment prediction in body fluid samples of non-small-cell lung cancer patients. J Exp Clin Cancer Res 30: 111, 2011.

22. Diaz LA Jr and Bardelli A: Liquid biopsies: Genotyping circulating tumor DNA. J Clin Oncol 32: 579-586, 2014.

23. Hindson BJ, Ness KD, Masquelier DA, Belgrader P, Heredia NJ, Makarewicz AJ, Bright IJ, Lucero MY, Hiddessen AL, Legler TC, et al: High-throughput droplet digital PCR system for absolute quantitation of DNA copy number. Anal Chem 83: 8604-8610, 2011.

24. Chen JD, Yang HI, Iloeje UH, You SL, Lu SN, Wang LY, Su J, Sun CA, Liaw YF and Chen CJ; Risk Evaluation of Viral Load Elevation and Associated Liver Disease/Cancer in HBV (REVEAL-HBV) Study Group: Carriers of inactive hepatitis $B$ virus are still at risk for hepatocellular carcinoma and liver-related death. Gastroenterology 138: 1747-1754, 2010.

25. Morisset D, Štebih D, Milavec M, Gruden K and Žel J: Quantitative analysis of food and feed samples with droplet digital PCR. PLoS One 8: e62583, 2013.

26. Miotke L, Lau BT, Rumma RT and Ji HP: High sensitivity detection and quantitation of DNA copy number and single nucleotide variants with single color droplet digital PCR. Anal Chem 86: 2618-2624, 2014

27. Beck J, Bierau S, Balzer S, Andag R, Kanzow P, Schmitz J, Gaedcke J, Moerer O, Slotta JE, Walson P, et al: Digital droplet PCR for rapid quantification of donor DNA in the circulation of transplant recipients as a potential universal biomarker of graft injury. Clin Chem 59: 1732-1741, 2013.

28. Heredia NJ, Belgrader P, Wang S, Koehler R, Regan J, Cosman AM, Saxonov S, Hindson B, Tanner SC, Brown AS and Karlin-Neumann G: Droplet Digital ${ }^{\mathrm{TM}}$ PCR quantitation of HER2 expression in FFPE breast cancer samples. Methods 59: S20-S23, 2013.

29. Zhang Y, Xu Y, Zhong W, Zhao J, Chen M, Zhang L, Li L and Wang M: Total DNA input is a crucial determinant of the sensitivity of plasma cell-free DNA EGFR mutation detection using droplet digital PCR. Oncotarget 8: 5861-5873, 2017. 
30. Ahn MJ, Lee JY, Lim SH, Lee MY, Kim H, Kim HK, Byeon S, Ham JS, Yali B, Xiumin W, et al: Dynamic serial monitoring of EGFR mutations in plasma DNA samples in EGFR mutant NSCLC patients treated with EGFR TKI. J Clin Oncol 33: 8078, 2015.

31. Watanabe M, Kawaguchi T, Isa S, Ando M, Tamiya A, Kubo A, Saka H, Takeo S, Adachi H, Tagawa T, et al: Ultra-Sensitive detection of the pretreatment EGFR T790M mutation in non-small cell lung cancer patients with an EGFR-activating mutation using droplet digital PCR. Clin Cancer Res 21: 3552-3560, 2015.

32. Guo SL, Chen XZ, Xiao YZ, Zhu SQ, Gong YQ and Yang JP Use of the duplex TaqMan MGB probe for simultaneous detection of Perkinsus and Bonamia in marine shellfish. J Appl Oceanography 33: 284-289, 2014.

33. Chang S, Chen W and Yang J: Another formula for calculating the gene change rate in real-time RT-PCR. Mol Biol Rep 36: 2165-2168, 2009.

34. Grabe T, Lategahn J and Rauh D: C797S Resistance: The undruggable EGFR mutation in non-small cell lung cancer? ACS Med Chem Lett 9: 779-782, 2018.

35. Greenlee RT, Hill-Harmon MB, Murray T and Thun M: Cancer statistics, 2001. CA Cancer J Clin 51: 15-36, 2001.

36. Mao C, Yuan JQ, Yang ZY, Fu XH, Wu XY and Tang JL: Blood as a substitute for tumor tissue in detecting EGFR mutations for guiding EGFR TKIs treatment of nonsmall cell lung cancer: A systematic review and meta-analysis. Medicine (Baltimore) 94 e775, 2015.
37. Gonzalez de Castro D, Angulo B, Gomez B, Mair D, Martinez R, Suarez-Gauthier A, Shieh F, Velez M, Brophy VH, Lawrence HJ and Lopez-Rios F: A comparison of three methods for detecting KRAS mutations in formalin-fixed colorectal cancer specimens. Br J Cancer 107: 345-351, 2012.

38. Kim SS, Choi HJ, Kim JJ, Kim MS, Lee IS, Byun B, Jia L, Oh MR, Moon Y, Park S, et al: Droplet digital PCR-based EGFR mutation detection with an internal quality control index to determine the quality of DNA. Sci Rep 8: 543, 2018.

39. Zhu Y, Guo Z, Liu Y, Zheng X, Yang G and Zheng G: A novel ARMS-based assay for the quantification of EGFR mutations in patients with lung adenocarcinoma. Oncol Lett 15: 2905-2912, 2018.

This work is licensed under a Creative Commons Attribution-NonCommercial-NoDerivatives 4.0 International (CC BY-NC-ND 4.0) License. 